\title{
Non-specific interstitial pneumonia as pulmonary involvement of systemic sclerosis
}

\author{
J Fujita, T Yoshinouchi, Y Ohtsuki, M Tokuda, Y Yang, I Yamadori, S Bandoh, T Ishida, \\ J Takahara, R Ueda
}

\begin{abstract}
The pathological features of lung disease in nine patients with systemic sclerosis (SSc) were evaluated. The patients comprised one man and eight women, with a median age of 58 years. SSc was diagnosed according to the criteria of the American Rheumatism Association. In all patients, high resolution computed radiographic scanning of the lungs (HRCT) was performed, and apparent honeycomb formation was seen in four patients. Pathologically, four patients were diagnosed with usual interstitial pneumonia (UIP), three with non-specific interstitial pneumonia (NSIP) group II, one NSIP group II-III, and one NSIP group II with diffuse alveolar damage. HRCT showed no apparent honeycomb formations in patients diagnosed with NSIP. This is the first report describing NSIP as a pulmonary complication of SSc.

(Ann Rheum Dis 2001;60:281-283)
\end{abstract}

First Department of Internal Medicine, Kagawa Medical University, Kagawa, Japan

J Fujita

Y Yang

$S$ Bandoh

M Tokuda

T Ishida

J Takahara

Department of Internal Medicine, Nagoya City

University, Nagoya, Japan

T Yoshinouchi

R Ueda

Department of Pathology, Okayama University Medical School, Okayama,

Japan

I Yamadori

Department of Pathology, Kochi Medical School, Kochi, Japan

Y Ohtsuki

Correspondence to: Dr J Fujita, First Department of Internal Medicine, Kagawa Medical University, 1750-1, Miki-cho, Kita-gun, Kagawa, 761-0793, Japan jiro@kms.ac.jp

Accepted 2 August 2000
It is well known that pulmonary fibrosis is often associated with systemic sclerosis (SSc). Pathological findings include extensive interstitial fibrosis with cystic changes, bronchiolectasis, and pleural thickening. ${ }^{1}$ In addition, alveolitis has also been seen in these studies, but it is uncertain whether the inflammatory cell infiltrate precedes, accompanies, or follows fibrosis of the alveolar walls. ${ }^{1}$

In 1991, Harrison et al reported detailed descriptions of antemortem lung disease in SSc, and they concluded that there are no qualitative or quantitative differences other than a higher prevalence of focal lymphoid hyperplasia in the patients with SSc than in the patients with lone cryptogenic fibrosing alveolitis (CFA). ${ }^{2}$ However, it has also been reported that the prognosis for pulmonary fibrosis associated with SSc is significantly better than that for patients with lone CFA. . $^{3-5}$

In 1994, Katzenstein and Fiorelli reported the histological features and clinical significance of non-specific interstitial pneumonia (NSIP). ${ }^{6}$ They reviewed 64 such cases, from which three histological patterns emerged. ${ }^{6}$ In $48 \%$ of the cases, pneumonia was characterised by a cellular interstitial infiltrate with little or no fibrosis (NSIP group I). This pattern probably corresponds to the "cellular interstitial pneumonia" described in some patients with rheumatoid arthritis ${ }^{7}$ and dermatomyositis. ${ }^{8}$ In $38 \%$ of cases, both inflammation and fibrosis were seen (NSIP group II). In the remaining $14 \%$ of cases, dense fibrosis was the dominant histological feature (NSIP group III). ${ }^{6}$
However, there are presently no reports which have re-evaluated pathological findings of pulmonary fibrosis associated with SSc since the report of NSIP. With this background, our present study describes the pathological classification of pulmonary fibrosis in nine patients with SSc.

\section{Materials and methods}

SUBJECTS

Between 1990 and 1999, nine patients with pulmonary fibrosis associated with SSc, in whom pathological evaluation of the lung was possible, were retrospectively entered into this study. All patients met preliminary criteria of the American Rheumatism Association for a diagnosis of SSc. ${ }^{9}$ Patients with other collagen diseases in addition to SSc were excluded. In all patients, high resolution computed radiographic scanning of the lungs (HRCT) was performed. This was carried out by making 12 slices from above the aortic arch to the diaphragm with $5 \mathrm{~mm}$ collimation in a bone detail algorithm during moderate inspiration. The diagnosis of pulmonary fibrosis was made on clinical, radiological, physiological, and histological grounds. No evidence was found that interstitial pneumonia was caused by drugs or recent acute lung injury. In addition, because there were no improvements of symptoms or radiological findings during the hospital stay, and no antibodies to Trichosporon cutaneum were demonstrated, we believe that patients with hypersensitivity pneumonitis were not included in our study.

\section{PATHOLOGICAL CRITERIA}

All pathological specimens were analysed by lung pathologists (IY and YO). Pathological classification of interstitial pneumonia was performed according to the definition reported by Liebow. ${ }^{10}$ The histological hallmark and chief diagnostic criterion of usual interstitial pneumonia (UIP) is a heterogeneous appearance at low magnification with alternating areas of normal lung, interstitial inflammation, fibrosis, and honeycomb change. ${ }^{11}$ NSIP was diagnosed according to the criteria for NSIP described by Katzenstein and Fiorelli. ${ }^{6}$ Briefly, NSIP is characterised by the presence of varying degrees of inflammation and fibrosis within the alveolar walls, but it lacks more specific changes that indicate a diagnosis of UIP, desquamative interstitial pneumonia, or acute interstitial pneumonia. Most examples contain either inflammation with minimal fibrosis or a mixture of inflammation and fibrosis, though a few are composed mainly of fibrosis with minimal inflammation. The process may be patchy 
Table 1 Patient characteristics and pathological findings of interstitial pneumonia associated with systemic sclerosis

\begin{tabular}{|c|c|c|c|c|c|c|c|}
\hline Patient No & Age & Sex & Autoantibody & Radiology & Honeycomb & Specimen & Pathological classification \\
\hline 1 & 58 & $\mathrm{~F}$ & Scl-70 & Bilateral reticulonodular & $(-)$ & Open lung biopsy & $\mathrm{NSIP}^{\star} \mathrm{II}$ \\
\hline 2 & 50 & M & $\mathrm{ND}^{\star}$ & Bilateral reticulonodular & Micro & Open lung biopsy & NSIP II-III \\
\hline 3 & 58 & $\mathrm{~F}$ & Rheumatoid factor & $\begin{array}{l}\text { Bilateral reticulonodular }+ \\
\text { ground glass (right middle) }\end{array}$ & $(-)$ & Open lung biopsy & NSIP II \\
\hline 4 & 53 & $\mathrm{~F}$ & SS-A (+) & Honeycomb + ground glass & $(+)$ & Open lung biopsy & $\mathrm{UIP}^{\star}$ \\
\hline 5 & 67 & $\mathrm{~F}$ & $\mathrm{ANA}^{\star}, \mathrm{Scl}-70$ & "Honeycomb" & $(+)$ & Necropsy & UIP \\
\hline 6 & 50 & $\mathrm{~F}$ & ND & Bilateral reticulonodular & $(-)$ & Necropsy & NSIP II \\
\hline 7 & 41 & $\mathrm{~F}$ & ANA, Scl-70 & "Honeycomb" & $(+)$ & Necropsy & UIP \\
\hline 8 & 59 & $\mathrm{~F}$ & ND & Bilateral ground glass & $(-)$ & Necropsy & NSIP II + diffuse alveolar damage \\
\hline 9 & 62 & $\mathrm{~F}$ & ND & "Honeycomb" & $(+)$ & Necropsy & UIP \\
\hline
\end{tabular}

${ }^{\star} \mathrm{ND}=$ not detected; $\mathrm{ANA}=$ antinuclear antibody; NSIP = non-specific interstitial pneumonia; UIP $=$ usual interstitial pneumonia.

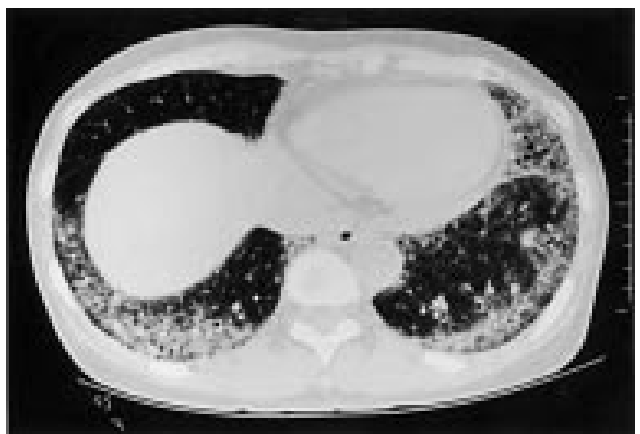

Figure 1 Chest computed tomography finding of patient 1 who was diagnosed with non-specific interstitial pneumonia group II.

with intervening areas of unaffected lung, but the changes are temporally uniform in that they appear to have occurred over a single, relatively narrow time span. This temporal uniformity contrasts sharply with the temporal heterogeneity characteristic of UIP. ${ }^{12}$

\section{Results}

Table 1 shows the characteristics of nine patients with SSc with lung fibrosis. The patients comprised one man and eight women, with a median age of 58 years (range $41-67$ ). No patients had histories of occupational inhalation of dust. No patients showed digital clubbing. In auscultatory findings, inspiratory fine crackles in the back lower lung fields were seen in all patients. In four patients the diagnosis was made by open lung biopsy. In the other five

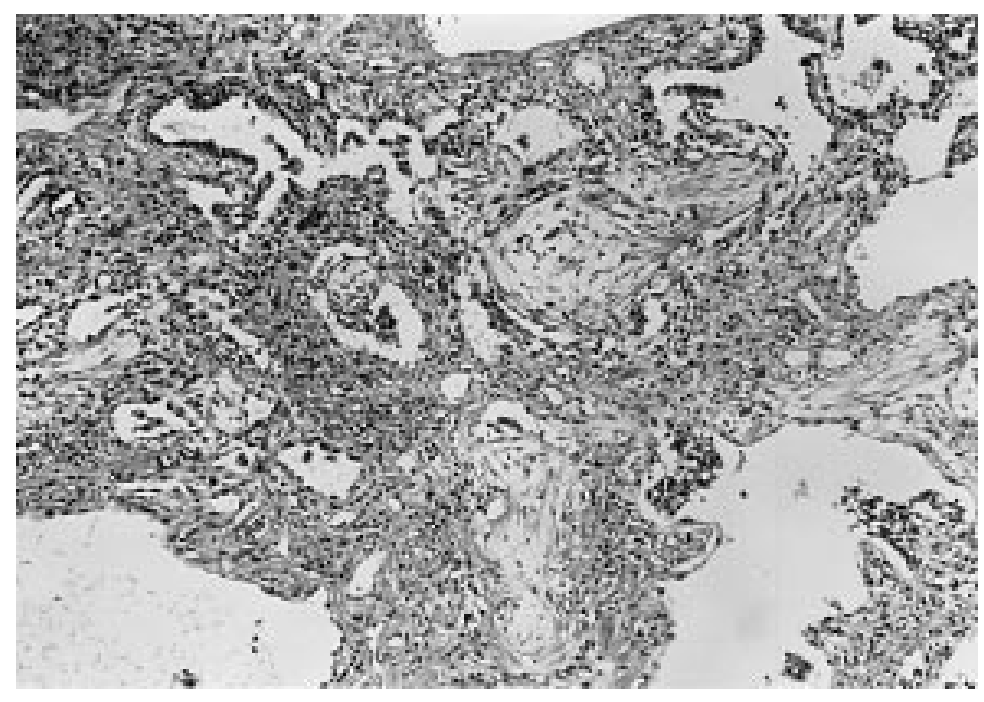

Figure 2 Lung biopsy specimen of patient 1 shows diffuse alveolar inflammation and abundant Masson's body formation in the alveolar spaces. patients, pathological classification was performed in lung specimens obtained at necropsy.

Table 1 also shows the predominant HRCT features of the nine patients. In four patients apparent honeycomb formation was seen. In one patient micro-honeycomb formation was seen. In the remaining four patients honeycomb formation was not seen with HRCT.

Histologically, four patients were diagnosed with UIP, three with NSIP group II, one with NSIP group II-III, and one with NSIP group II with diffuse alveolar damage (table 1). The patient with diffuse alveolar damage also had the complication of massive bronchopneumonia during the period on a ventilator. Therefore, we believe that this infection reflects the pathological finding of diffuse alveolar damage.

There was no apparent honeycomb formation on HRCT in five patients diagnosed with NSIP (table 1). Figure 1 shows chest computed tomography finding of patient 1 who was diagnosed with NSIP group II. Interstitial and patchy parenchymal opacifications in both lungs were seen, predominantly in the middle and lower lung zones. Scattered ground glass opacities were also the most common manifestation. However, there were no apparent honeycomb formations.

A lung biopsy specimen obtained from patient 1 showed diffuse alveolar inflammation and abundant Masson's body formation in the alveolar spaces (fig 2).

\section{Discussion}

In this study we demonstrate pathological features of nine patients with SSc with lung fibrosis, and this is the first report to show that in half the cases the lung fibrosis associated with SSc was NSIP.

NSIP was first described by Katzenstein and Fiorelli in $1994 .{ }^{6}$ In addition, Bjoraker et al reported that patients with UIP have worse survival than patients with other types of idiopathic chronic interstitial pneumonias, including NSIP. ${ }^{13}$ NSIP is not likely to be a single clinicopathological entity, and Katzenstein and Fiorelli found that $16 \%$ of patients had an associated collagen vascular disease, $17 \%$ had a history of inhalational exposure to noxious agents, and $8 \%$ had a history of recent surgery, severe pneumonia, or adult respiratory distress syndrome. ${ }^{6}$

In 1991 Harrison et al reported detailed descriptions of antemortem lung disease in SSc. ${ }^{2}$ They examined 46 biopsy specimens and reported that patchy inflammatory cell infiltra- 
tion of the interstitium and alveolar spaces occurs together with fibrosis of the alveolar walls from the earliest stages of the disease. In addition, they also reported that interstitial infiltration by lymphocytes and plasma cells was one of the earliest abnormalities. ${ }^{2}$ Although these pathological findings resemble that of NSIP, they concluded that the parenchymal abnormalities seen in lung biopsy samples from patients with SSc are indistinguishable from those of patients with lone CFA, with the exception of focal lymphoid hyperplasia, which is more common in the patients with SSc. ${ }^{2}$

However, despite their pathological identity, preliminary evidence suggests that patients with SSc with fibrosing alveolitis have a more favourable prognosis than those with lone $\mathrm{CFA}^{3-5}$ Therefore, it is important to evaluate factors that may be modulating the disease in SSc. Wells et al reported bronchoalveolar lavage cellularity in fibrosing alveolitis associated with SSc and they showed that lymphocyte percentages are higher in patients with fibrosing alveolitis associated with SSc than in patients with lone CFA. ${ }^{14}{ }^{15}$ In addition, Wells et al also evaluated the computed tomographic appearance in the fibrosing alveolitis associated in 27 patients with SSc and reported that a ground glass pattern predominated in three patients, a ground glass pattern and reticular pattern were equally extensive in 10 patients, and a reticular pattern was the most extensive abnormality in 14 patients. ${ }^{15}$ Although histological evaluation was not performed in these reports, these observations suggest that NSIP might have been included in their study group. Therefore, our findings may provide a reason why there is a better prognosis of pulmonary fibrosis associated with SSc than lone CFA.

In summary, our study shows that half of the patients with pulmonary fibrosis associated with SSc were diagnosed with NSIP, and this may explain the better prognosis of lung fibrosis associated with SSc than lone CFA.
1 Rossi GA, Bitterman PB, Rennard SI, Ferrans VJ, Crystal RG. Evidence for chronic inflammation as a component of the interstitial lung disease associated with progressive systemic sclerosis. Am Rev Respir Dis 1985;131:612-17.

2 Harrison NK, Myers AR, Corrin B, Soosay G, Dewar A, Black CM, et al. Structural features of interstitial lung disease in

3 Alton EW, Kwan S, Turner-Warwick M. Pulmonary involvement in scleroderma; comparison with cryptogenic fibrosing alveolitis. Thorax 1986;41:714P.

4 Wells AU, Cullinan P, Hansell DM, Rubens MB, Black CM, Newman-Taylor AJ, et al. Fibrosing alveolitis associated with systemic sclerosis has a better prognosis than lone cryptogenic fibrosing alveolitis. Am J Respir Crit Care Med cryptogenic fibrosin

5 Wells AU, Hansell DM, Rubens MB, Cailes JB, Black CM, du Bols RM. Functional Impairment in lone cryptogenic fibrosing alveolitis and fibrosing alveolitis associated with systemic sclerosis: a comparison. Am J Respir Crit Care Med 1997;155:1657-64

6 Katzenstein A, Fiorelli RF. Nonspecific interstitial pneumonia/fibrosis. Histologic features and clinical significance. Am J Surg Pathol 1994;18:136-47.

7 Tazelaar HD, Viggiano RW, Pickersgill J, Colby TV. Interstitial lung disease in polymyositis and dermatomyositis: clinical features and prognosis as correlated with histologic findings. Am Rev Respir Dis 1990;141:727-33.

8 Yousem SA, Colby TV, Carrington CB. Lung biopsy in rheumatoid arthritis. Am Rev Respir Dis 1985;131:770-7.

9 Subcommittee for scleroderma criteria of the American Rheumatism Association Diagnostic and Therapeutic Criteria Committee. Preliminary criteria for the classification of systemic sclerosis (scleroderma). Arthritis Rheum 1980; 23:581-90.

10 Liebow A. Definition and classification of interstitial pneumonias in human pathology. Progress in Respiratory Research 1975;8:1.

11 American Thoracic Society. Idiopathic pulomnary fibrosis: diagnosis and treatment. International Consensus Statement. American Thoracic Society (ATS) and the European Respiratory Society (ERS). Am J Respir Crit Care Med 2000;161:646-64.

12 Katazenstein AA, Myers JL. Idiopathic pulmonary fibrosis. Clinical relevance of pathologic classification. Am J Respir Crit Care Med 1998;157:1301-15.

13 Bjoraker JA, Ryu JH, Edwin MK, Myers JL, Tazalaar HD, Schroeder DR, et al. Prognostic significance of histopathologic subsets in idiopathic pulmonary fibrosis. Am J Respir Crit Care Med 1998;157:199-203.

14 Wells AU, Hansell DM, Haslam PL, Rubens MB, Cailes J, Black CM, et al. Bronchoalveolar lavage cellularity: lone cryptogenic fibrosing alveolitis compared with the fibrosing alveolitis of systemic sclerosis. Am J Respir Crit Care Med 1998;157:1474-82.

15 Wells AU, Hansell DM, Rubens MB, Cullinan P, Haslam PL, Black CM, et al. Fibrosing alveolitis in systemic sclerosis: bronchoalveolar lavage findings in relation to computed tomographic appearance. Am J Respir Crit Care Med 1994;150:462-8. 